\title{
Orbital apex cyst: a rare cause of compressive optic neuropathy post-functional endoscopic sinus surgery
}

\author{
Yi Ni Koh ${ }^{1,2}$ \\ Shu Fen $\mathrm{Ho}^{2}$ \\ Letchumanan Pathma ${ }^{3}$ \\ Harvinder Singh ${ }^{3}$ \\ Embong Zunaina' \\ 'Department of Ophthalmology, \\ School of Medical Sciences, Universiti \\ Sains Malaysia, Kubang Kerian, \\ Kelantan, Malaysia; ${ }^{2}$ Department \\ of Ophthalmology, ${ }^{3}$ Department \\ of Otorhinolaryngology, Hospital \\ Raja Permaisuri Bainun, Ipoh, Perak, \\ Malaysia
}

This article was published in the following Dove Press journal: International Medical Case Reports Journal 28 July 2017

Number of times this article has been viewed

\begin{abstract}
There are various causes that can lead to compressive optic neuropathy. We present here orbital apex cyst as an unusual cause of compressive optic neuropathy in a 49-year-old male. He presented with 2 weeks painless loss of vision in the left eye with left-sided headache. He had had left functional endoscopic sinus surgery for left nasal polyps 4 years earlier. Magnetic resonance imaging of brain and orbit revealed a left discrete orbital nodule, possibly orbital cyst or mucocele, which was compressing on the left optic nerve. Left eye vision improved markedly from hand movement to $6 / 36$ pinhole $6 / 18$ after initiation of intravenous dexamethasone. A subsequent endoscopic endonasal left optic nerve decompression found the orbital nodule lesion to be an orbital cyst. Marsupialization was performed instead of excision, as the cyst ruptured intraoperatively. Postoperative vision improved to $6 / 7.5$ with normal optic nerve function postoperatively. Possible cause of orbital apex cyst is discussed.
\end{abstract}

Keywords: orbital cyst, compressive optic neuropathy, functional endoscopic sinus surgery

\section{Introduction}

Orbital cysts are a rare pathological entity that can be divided into either primary (congenital) or secondary cysts. ${ }^{1}$ Primary lesions are choristomas that are formed by embryological maldevelopment of a tissue type that is normally not found at that site. ${ }^{2}$ Secondary orbital cysts result from secondary implantation of surface epithelium, usually after trauma or surgery. ${ }^{1}$ Those located in the orbit may arise from cutaneous, conjunctival, respiratory, or lacrimal gland epithelium.

We report a case of compressive optic neuropathy secondary to orbital apex cyst in a patient post-functional endoscopic sinus surgery (FESS).

\section{Case report}

A 49-year-old gentleman with no known medical illness presented with left eye acute painless central scotoma for 2 weeks. There was no metamorphopsia, floaters, or flashes of light. The central scotoma was associated with left-sided headache. However, there was no nausea or vomiting. He had a history of left FESS for left nasal polyps 4 years earlier. Otherwise, there was no history of recent trauma or ocular surgery. He denied a history of limb weakness or altered consciousness.

Ocular assessment revealed visual acuity of hand movement in the left eye and 6/9 in the right eye. Left optic nerve function was impaired with positive relative afferent pupillary defect. There was no proptosis or globe displacement seen with normal extraocular movement. Anterior segment examination was normal bilaterally. The left
Correspondence: Yi Ni Koh

Department of Ophthalmology, School of Medical Sciences, Universiti Sains Malaysia, 16150 Kubang Kerian, Kelantan, Malaysia

Tel +6097676362

Fax +6097653370

Email kohyini@hotmail.com 
A

Single Field Analysis

Central 24-2 Threshold Test

Fixation Monitor: Gaze/Blind Spot

Fixation Target: Central

Fixation Losses: 0/11

False POS Erros: $3 \%$

False NEG Errors: N/A

Test Duration: 05:36

Fovea: $<\mathrm{O} d \mathrm{~B}$
Stimulus: III, White

Background: 31.5 ASB

Strategy: SITA-Fast
Eye: Left

$\begin{array}{lll}\text { Pupil Diameter: } 6.2 \mathrm{~mm} & \text { Date: } 01-12-2015 \\ \text { Visual Acuity: } & \text { Time: } 6: 41 \mathrm{AM} \\ \text { RX: DS DC X } & \text { Age: } 49\end{array}$

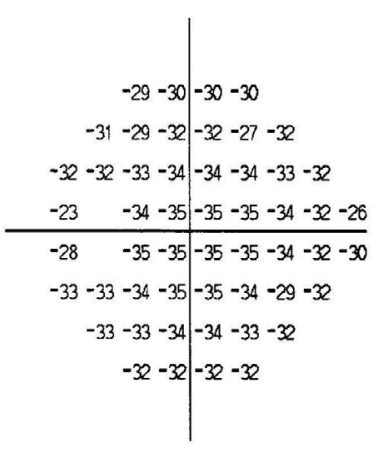

Total Deviation

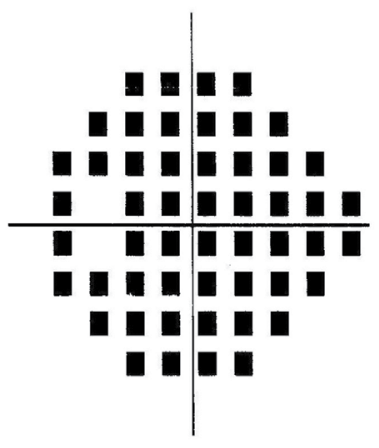

Pattern Deviation not shown for severely depressed fields. Refer to Total Deviation.

Pattern Deviation not shown for severely depressed fields. Refer to Total Deviation.

\begin{abstract}
Pattern Deviation
\end{abstract}

$$
\begin{aligned}
& ::<5 \% \\
& \not 2<2 \% \\
& \text { 苝 }<1 \% \\
& <0.5 \%
\end{aligned}
$$

GHT

Outside normal limits

$$
\begin{array}{ll}
\text { VFI } & 1 \% \\
\text { MD } & -32.63 \mathrm{~dB} \quad \mathrm{P}<0.5 \% \\
\text { PSD } & 2.54 \mathrm{~dB} \quad \mathrm{P}<2 \%
\end{array}
$$

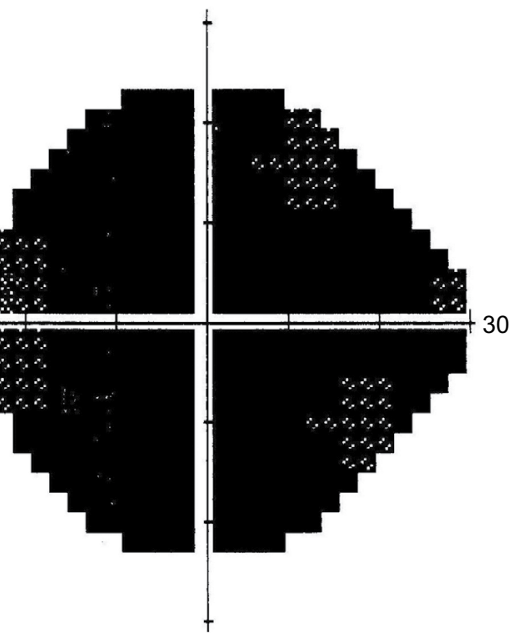


B

Single Field Analysis

Central 24-2 Threshold Test

Fixation Monitor: Gaze/Blind Spot

Fixation Target: Central

Fixation Losses: 0/11

False POS Erros: $0 \%$

False NEG Errors: $3 \%$

Test Duration: 03:20

Fovea: $37 \mathrm{~dB}$
Stimulus: III, White Background: 31.5 ASB Strategy: SITA-Fast
Eye: Right

Pupil Diameter: $5.2 \mathrm{~mm}$

Visual Acuity:

RX: DS DC $X$

Time: 6:36 AM

Age: 49

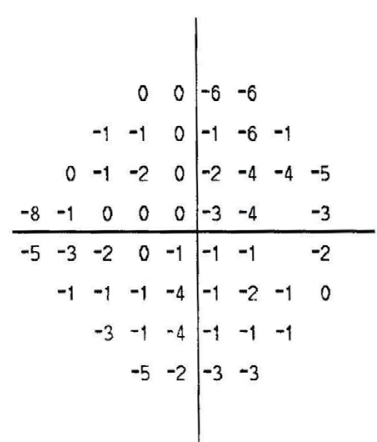

Total Deviation

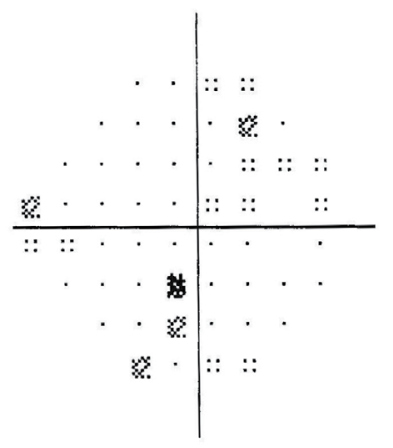

30 \begin{tabular}{llllllllll}
20 & 30 & 32 & 33 & 33 & 29 & 29 & 27 & 27 \\
\cline { 2 - 7 } & 23 & 27 & 30 & 33 & 32 & 33 & 32 & 40 & 28
\end{tabular}

29

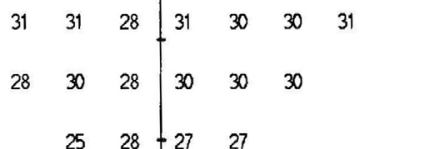

$+$

\begin{tabular}{ll|lll}
29 & 30 & 29 & 24 & 28
\end{tabular}

$f^{27}$

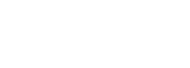

$\begin{array}{lllll}0 & 0 & -5 & -6\end{array}$

\begin{tabular}{lll|lll}
0 & -1 & 0 & -1 & -5 & -1
\end{tabular}

$\begin{array}{llllllll}0 & -1 & -2 & 0 & -2 & -4 & -4 & -5\end{array}$

\begin{tabular}{ccccc|ccc}
-8 & -1 & 0 & 0 & 0 & -3 & -3 & -3 \\
\hline-5 & -3 & -2 & 0 & -1 & 0 & -1 & -2
\end{tabular}

$\begin{array}{llllllll}-1 & 0 & -1 & -4 & -1 & -2 & -1 & 0\end{array}$

$\begin{array}{lllllll}-3 & -1 & -4 & -1 & -1 & -1\end{array}$

$\begin{array}{llll}-5 & -2 & -3 & -3\end{array}$

(2)

Pattern Deviation

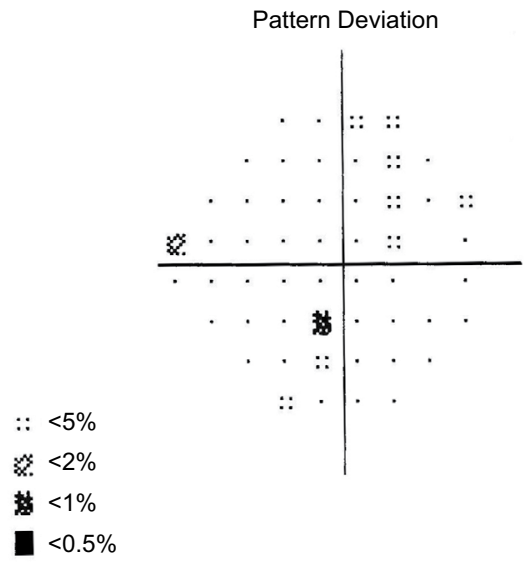

GHT

Within normal limits

VFI $98 \%$

MD $\quad-1.88 \mathrm{~dB} P<5 \%$

PSD $\quad 1.80 \mathrm{~dB} P<10 \%$

Figure I Humphrey visual field test showed normal visual field in the right eye (A) and diffuse depression of visual field in the left eye (B)

Abbreviations: GHT, glaucoma hemifield test; MD, mean defect; NEG, negative; POS, positive; PSD, pattern standard deviation. 
fundus showed normal retina with no macular edema or any macular abnormalities. The left optic disc was pink in color, with a well-defined margin and normal cup-disc ratio. The fundus examination and optic disc of the fellow eye were normal. Visual field test showed a normal right visual field and generalized depression of left visual field with a mean defect (MD) of -32.63 decibels (dB) (Figure 1).

Magnetic resonance imaging (MRI) of brain and orbit were performed to rule out any space-occupying lesion or any optic nerve compressive lesion. MRI of brain and orbit showed presence of a discrete orbital nodule measuring $14.77 \times 12.58 \mathrm{~mm}$ at the left orbital apex (Figure 2). The orbital nodule was compressing the superior rectus muscle and optic nerve. It demonstrated low T1 signal and high T2 signals in MRI. There was no enhancement seen with contrast. There was an incidental finding of right ethmoidal cystic lesion, and possibly a mucocele was noted in this imaging. Radiologically, the left orbital nodule that was compressing on the left optic nerve was suggestive of a mucocele or orbital cyst. There was no intracranial or extraorbital tissue extension or any bony erosion surrounding the lesion. However, computed tomography (CT) of paranasal sinuses showed a small bony defect seen at the wall between the lesion and left sphenoid sinus, but no obvious extension of this lesion into the left sphenoid sinus was seen. Based on clinical assessment and imaging findings, a diagnosis of left compressive optic neuropathy secondary to discrete orbital nodule was made.

In view of the compressive lesion of the optic nerve, the patient was comanaged with the otorhinolaryngology team. The patient was started on intravenous dexamethasone $8 \mathrm{mg}$ every 8 hours to reduce the possible inflammation of the lesion. Left eye vision improved markedly from hand movement to $6 / 36$ pinhole $6 / 18$ the next day. However, it did not resolve completely with medical treatment. Subsequently, after 4 days of dexamethasone, the patient underwent endoscopic endonasal decompression of the left optic nerve by the otorhinolaryngology team. Intraoperatively, the orbital nodule lesion was found to be an orbital cyst. The orbital cyst ruptured during manipulation and released a yellowish fluid. Due to tight orbital space, marsupialization was performed instead of excision of the cyst. No specimen from the orbital lesion was sent for histological examination. Postoperatively, left visual acuity improved to $6 / 7.5$ with normal optic nerve function. There was improvement of visual field assessment with residual inferior visual field defect (Figure 3).

The previous CT of paranasal sinuses prior to FESS 4 years earlier was retrieved and showed no orbital lesion

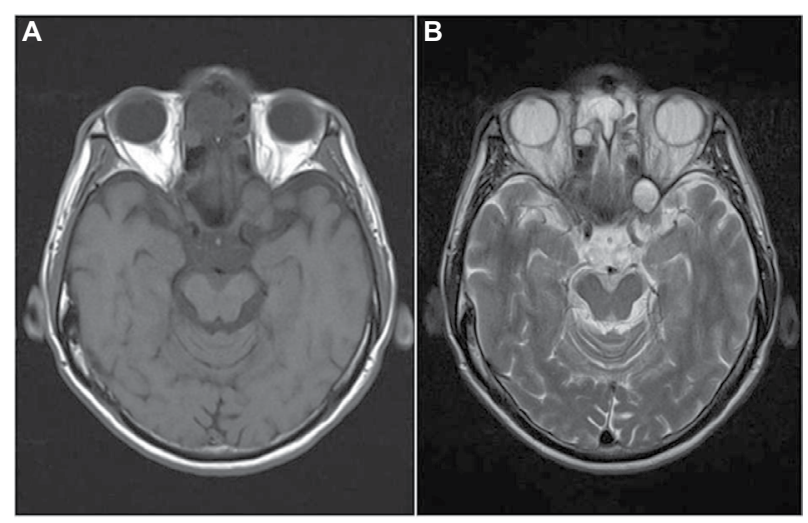

Figure 2 MRI of brain and orbits showed a discrete nodule of low TI (A) and high T2 (B) signals at the left orbital apex, compressing the superior rectus muscle and optic nerve. Incidental finding of right ethmoidal cystic lesion; possibly, a mucocele was noted in this imaging.

Abbreviation: MRI, magnetic resonance imaging.

at the left orbital apex (Figure 4). Finally, a diagnosis of compressive optic neuropathy secondary to orbital apex cyst post-FESS was made in this patient. A 1-year follow-up postsurgery showed no evidence of recurrence.

\section{Consent}

Written informed consent was obtained from the patient for publication of this case report and any accompanying images.

\section{Discussion}

Orbital cystic lesions may be subdivided into developmental anomalies and acquired lesions. These include dermoid cysts, epidermoid cyst, colobomatous cysts, epithelial and appendage cysts, cephaloceles, and mucoceles. Respiratory epithelial cysts, mucoceles, and epidermoid cysts are among the orbital cystic lesions that are associated with paranasal sinus disease, orbital trauma, or surgery. Orbital cysts lined by respiratory epithelium, for instance, mucoceles and respiratory epithelial cysts, are commonly secondary to chronic paranasal sinus disease, and sometimes due to sinus trauma many years earlier. Rarely, primary orbital cysts lined by respiratory epithelium may occur without extension from the nasal cavity or paranasal sinuses. ${ }^{1-3}$

In this case, orbital cystic lesion was not seen in the previous $\mathrm{CT}$ of the paranasal sinuses, which was done prior to FESS many years earlier. This may suggest the possibility of implantation of surface epithelium during FESS, which eventually caused the development of secondary orbital cyst, as seen in this case. Unfortunately, there was no histological result to confirm the type of orbital cyst. 
Single Field Analysis

Central 30-2 Threshold Test

Fixation Monitor: Blind Spot

Fixation Target: Central

Fixation Losses: 0/15

False POS Erros: $0 \%$

False NEG Errors: $0 \%$

Test Duration: 05:49

Fovea: $26 \mathrm{~dB}$
Stimulus: III, White

Background: 31.5 ASB

Strategy: SITA-Fast
Eye: Left

Pupil Diameter: Date: 09-12-2015

Visual Acuity: Time: $5.42 \mathrm{AM}$

RX: DS DC $X \quad$ Age: 49

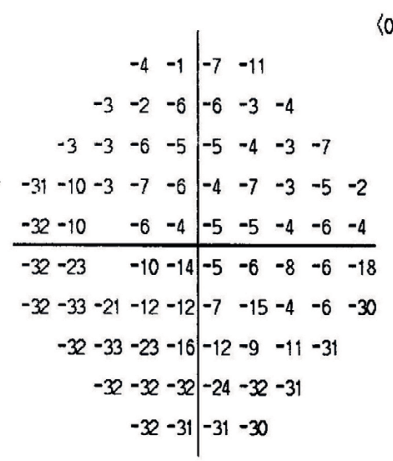

Total Deviation

.. $\mid:$ 㷊

. $\quad: \quad \%$

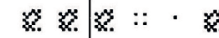

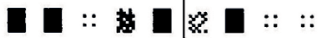

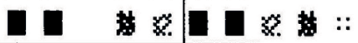

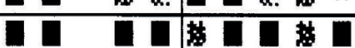

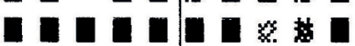

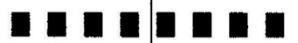

口曰

$\square \mathbf{\square}$
$::<5 \%$

\% $<2 \%$

$<<1 \%$

$<0.5 \%$ \begin{tabular}{ll|lll}
0 & 2 & -3 & -7
\end{tabular}

$\begin{array}{lllllll}1 & 2 & -2 & -2 & 0 & 0\end{array}$

$\begin{array}{llllllll}1 & 1 & -3 & -1 & -2 & 0 & 1 & -3\end{array}$

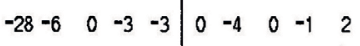

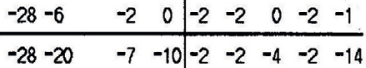

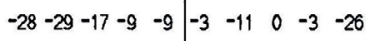

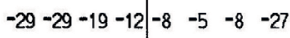

$-29-29-29-20-28-27$

$-28-28 \mid-27-27$

GHT

Outside normal limits

Pattern Deviation

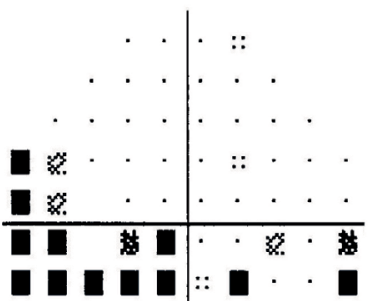

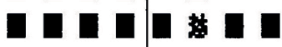

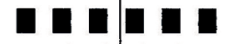

무

Figure 3 Humphrey visual field test showed improvement of visual field depression with residual inferior field defect of the left eye postoperatively. Abbreviations: GHT, glaucoma hemifield test; MD, mean defect; NEG, negative; POS, positive; PSD, pattern standard deviation. 


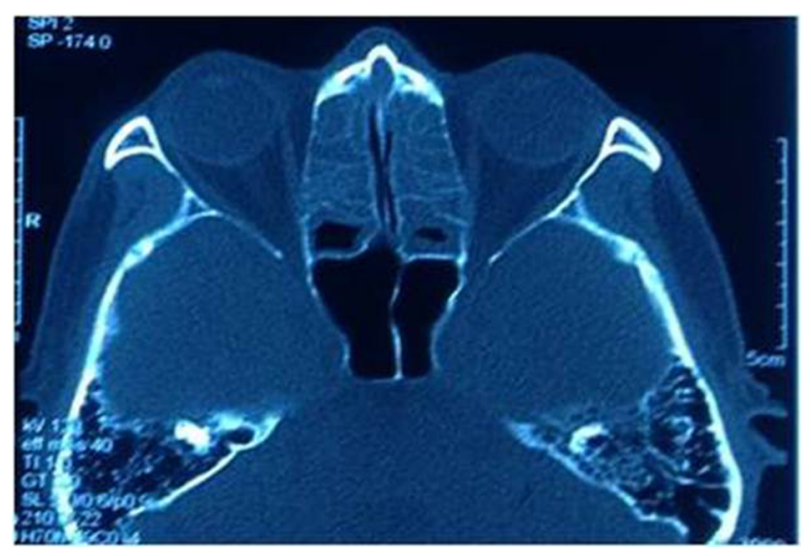

Figure 4 Contrast-enhanced CT of paranasal sinuses done prior to functional endoscopic sinus surgery, showed no orbital cystic lesion at the left orbital apex. Abbreviation: CT, computed tomography.

Clinical presentation varies according to the site and size of these lesions. Acute unilateral visual loss associated with retrobulbar optic nerve dysfunction usually suggests a diagnosis of ischemic optic neuropathy or optic neuritis. However, in rare instances, compressive optic neuropathy presumably from hemorrhage or from sudden interruption of the vascular supply to the optic nerve may cause acute monocular visual loss, which may mimic optic neuritis or ischemic optic neuropathy. ${ }^{4,5}$ Absence of cardiovascular risk factors and altitudinal field defects commonly found in ischemic optic neuropathy led us to investigate further, in this case, with imaging studies.

Imaging studies such as CT and MRI may aid in making preoperative diagnoses. As epidermoid tumors lack vascularity and do not enhance on CT, they will demonstrate low T1 signal and high T2 signals in MRI. Since epidermoids and cerebrospinal fluid have similar MRI signal characteristics, arachnoid cysts will be an important differential. Fluidattenuated inversion recovery (FLAIR) sequences allow for differentiation as to which epidermoids become hyperintense on FLAIR MRI, while arachnoid cysts will follow cerebrospinal fluid signal on FLAIR sequences. ${ }^{6}$

Management of optic nerve compressive lesions must be tailored according to the patient and the specific disease process. However, generally, the compression of the optic nerve must be relieved either by reducing the lesion's size medically and/or by removal of the lesion surgically. Despite the lack of radiological evidence of inflammation preoperatively in this case, high-dose intravenous dexamethasone appeared to improve vision, presumably by reducing the inflammation of the lesion and thus reducing the optic nerve compression prior to surgical intervention. On the other hand, endoscopic endonasal optic nerve decompression provides excellent visualization and unparalleled access to the tumor with minimal morbidity. A combination of high-dose intravenous dexamethasone and surgical intervention may improve the patient's visual prognosis. Ahmed et al ${ }^{1}$ suggested that orbital epidermoid cysts usually warrant surgical intervention and should be totally removed as they tend to recur with lipogranuloma formation, and because there is a remote possibility of malignant transformation. Goh et $\mathrm{al}^{3}$ recommended that complete surgical removal is necessary to prevent future recurrence. In this case, the orbital cyst's location at the orbital apex made the total excision of the cyst via endoscopic endonasal approach more difficult to perform due to the very limited operating field. A 1-year endoscopic review showed no recurrence, and further follow-up is planned.

Orbital apex cyst is a rare cause of compressive optic neuropathy. Although rare, proper diagnosis and prompt management can be sight saving. The administration of dexamethasone followed by surgical decompression allows for successful treatment of this condition with minimal morbidity.

The major limitation of this report is the lack of histologic evidence, as the diagnosis cannot be verified without a biopsy. Another limitation is that complete removal of the cyst should be done to limit the risk of recurrence and potential malignant transformation.

\section{Disclosure}

The authors report no conflicts of interest in this work.

\section{Reference}

1. Ahmed RA, Eltanamly RM. Orbital epidermoid cysts: a diagnosis to consider. J Ophthalmol. 2014;2014:508425.

2. Kanski JJ, Bowling B. Clinical Ophthalmology: A systematic approach. 7th ed. Philadelphia: Elsevier/Saunders; 2011:100-101.

3. Goh RL, Hardy TG, Williams RA, McNab AA. Respiratory epithelial cysts of the orbit. Orbit. 2016;35(5):245-249.

4. Lee AG, Lin DJ, Kaufman M, Golnik KC, Vaphiades MS, Eggenberger E. Atypical features prompting neuroimaging in acute optic neuropathy in adults. Can J Ophthalmol. 2000;35(6):325-330.

5. Lin MC, BeeYS, Sheu SJ. A suprasellar meningioma simulating atypical retrobulbar optic neuritis. J Chin Med Assoc. 2003;66(11):689-692.

6. Warakaulle DR, Anslow P. Differential diagnosis of intracranial lesions with high signal on T1 or low signal on T2-weighted MRI. Clin Radiol. 2003;58(12):922-933. 
The International Medical Case Reports Journal is an international, peer-reviewed open-access journal publishing original case reports from all medical specialties. Previously unpublished medical posters are also accepted relating to any area of clinical or preclinical science. Submissions should not normally exceed 2,000 words or
4 published pages including figures, diagrams and references. The manuscript management system is completely online and includes a very quick and fair peer-review system, which is all easy to use. Visit $\mathrm{http}: / /$ www.dovepress.com/testimonials.php to read real quotes from published authors.

Submit your manuscript here: https://www.dovepress.com/international-medical-case-reports-journal-journal 